\title{
Fast Image Super-resolution with Sparse Coding
}

\author{
Zhi-chao Yuan ${ }^{1, a}$, Ben-tu Li ${ }^{1}$
}

${ }^{1}$ Shandong Vocational College of Science and Technology, Weifang, 261053, China;

\begin{abstract}
In this paper, we introduce a novel fast image reconstruction method for super-resolution (SR) base on sparse coding. This method combine online dictionary learning and a fast sparse coding way, both of which can improve the efficiency of the reconstruction process and ensure the image visual quality. The new online optimization algorithm for dictionary learning based on stochastic approximations, which can drastically advance the learning speed, especially on millions of training samples. Meanwhile, we trained a neural network to speed up the reconstruction process, which based on iterative shrinkage-thresholding algorithm (ISTA), we called learned iterative shrinkage-thresholding algorithm (LISTA). It would produce best approximation sparse code with some fixed depth. We demonstrate that our approach can simultaneously improve the image fidelity and cost less computation.
\end{abstract}

Keywords: SRIR; sparse coding; super-resolution; fast image super-resolution.

\section{Introduction}

High-resolution image is valuable in many social areas. Such as reconstruct the high-resolution image from low-resolution image which take in Criminal Investigation, get high-accurate CT, MIR, Ultrasonic wave to help doctor diagnose disease. Additionally, this is also play an important role in HDTV, Video Rip and Military occasion.

The main contribution of this paper is combining the online dictionary learning and approximating sparse coefficient together to reconstruct the High-resolution image, so that the reconstruction rate is splendidly speedup. Learning the dictionary by online method process one element of the millions of training samples at a time, which based on stochastic approximations(Aharon \& Elad, 2008). Instead of optimize the 11-norm optimization for accurate sparse coefficients,

$$
f_{n}(\tilde{\mathrm{D}}) \triangleq \frac{1}{n} \sum_{i=1}^{n} l\left(\tilde{y}_{i}, \tilde{\mathrm{D}}\right) \text {, where } l\left(\tilde{y}_{i}, \tilde{\mathrm{D}}\right) \triangleq \min _{\alpha \in R^{k}} \frac{1}{2}\left\|\tilde{y}_{i}-\tilde{D} \alpha_{i}\right\|_{2}^{2}+\lambda\left\|\alpha_{i}\right\|_{1}
$$


As point by Bettou and Bousquet(Bousquet \& Bottou, 2007), maybe its not important in a perfect minimization of energy

$$
f(\tilde{D}) \triangleq E_{x}\left[l\left(\tilde{y}_{i}, \tilde{\mathrm{D}}\right)\right]=\lim _{n \rightarrow \infty} f_{n}(\tilde{D})
$$

The outline of our algorithm is described in algorithm 1

Algorithm1 Online dictionary learning

$$
\text { Require: } \quad x \in R^{m}: \mathrm{p}(x) \quad \text { (random }
$$

variable and an algorithm to draw i.i.d samples of $\mathrm{p}$ ), $\lambda \in R \quad$ (regularization parameter), $D_{0} \in R^{m \times k}$ (initial dictionary). $\mathrm{T}$ (number of iterations)

1: $A_{0} \leftarrow 0, B_{0} \leftarrow 0$ (reset the "past" function $f_{n}(\mathrm{D})$, but interested in expected minimization cost:
3: Draw $\mathrm{xt}$ from $\mathrm{p}(\mathrm{x})$. coding: compute using LARS

$$
\begin{gathered}
\alpha_{t}=\underset{\alpha \in R^{k}}{\arg \min } \frac{1}{2}\left\|x_{t}-D_{t-1} \alpha\right\|_{2}^{2}+\lambda\|\alpha\|_{1} \\
5: A_{t} \leftarrow A_{t-1}+\alpha_{t} \alpha_{t}^{T} ; \\
B_{t} \leftarrow B_{t-1}+x_{t} \alpha_{t}^{T} .
\end{gathered}
$$

7: Compute Dt using Algorithm 2, with $D_{t-1}$ as warm restart. So that information); 2 : For $\mathrm{t}=1$ to $\mathrm{T}$ do

$$
D_{t} \triangleq \underset{D \in C}{\arg \min } \frac{1}{t} \sum_{i=1}^{t} \frac{1}{2}\left\|x_{i}-D \alpha_{i}\right\|_{2}^{2}+\lambda\left\|\alpha_{i}\right\|_{1}=\underset{D \in C}{\arg \min } \frac{1}{t}\left(\frac{1}{2} \operatorname{Tr}\left(D^{T} D A_{t}\right)-\operatorname{Tr}\left(D^{T^{T} B_{t}}\right)\right)
$$

8: End for; 9: Return DT (learned dictionary).

We supposed the training set is composed of i.i.d samples of a distribution $\mathrm{p}(\mathrm{x})$, every loop it draws one element from the training set. It alternatively solve $\alpha$ by classical sparse coding and $\mathrm{D}$ by minimizing the following function:

$$
\bar{f}_{t}(D) @-\frac{1}{t} \sum_{i=1}^{t} \frac{1}{2}\left\|x_{i}-D \alpha_{i}\right\|_{2}^{2}+\left\|\alpha_{i}\right\|_{1}
$$

The quadratic function $f_{t}(\bar{D})$ is a surrogate. This function aggregates the previous information by the process of computing $\alpha$. The convergence analysis has shown that $\bar{f}_{t}(D)$ and $f_{t}$ convergence almost to the same limit(Mairal et al., 2009) . Because of $f_{t}(D)$ is close to $f_{t}$, so it can be efficiently computed via $D_{t-1}$ as warm restart.

Algorithm 2 Dictionary update

Require: $D=\left[\begin{array}{lllll}d_{1} & d_{2} & \mathrm{~K} & d_{k-1} & d_{k}\end{array}\right] \in R^{m \times k} \quad$ (input dictionary),

$$
A=\left[\begin{array}{llll}
a_{1} & a_{2} & \mathrm{~K} & a_{k}
\end{array}\right] \in R^{k \times k}=\sum_{i=1}^{t} \alpha_{i} \alpha_{i}^{T}, B=\left[\begin{array}{llll}
b_{1} & b_{2} & \mathrm{~K} & b_{k}
\end{array}\right] \in R^{m \times k}=\sum_{i=1}^{t} x_{i} \alpha_{i}^{T},
$$

1: Repeat;2: For $\mathrm{j}=1$ to $\mathrm{k}$ do

3: Update the $\mathrm{j}-1$ column to optimize for eqn. :

$$
u_{j} \leftarrow \frac{1}{A_{j j}}\left(b_{j}-D \alpha_{j}\right)+d_{j}, d_{j} \leftarrow \frac{1}{\max \left(\left\|u_{j}\right\|_{2}, 1\right)} u_{j}
$$

4: End for;5: Until convergence;6: Return D (update dictionary).

The algorithm 2 uses block-coordinate descent with warm restart to update the dictionary. The benefits of this method is parameter-free and does not require any learning rate tuning. It sequentially update D column by column when update the $\mathrm{j}$-th column, when set the other ones fixed under constraint $d_{j} d_{j}^{T} \leq 1$. Recent works shows that separable constraint could make the function convergence to a global optimum. Since we use Dt-1 to warm restart Dt, so the block-coordinate descent will more efficient. 


\subsection{Approximation sparse coefficient}

In this section, we employ the same neural network structure in paper(Gregor \& LeCun, 2010) to reconstruct the HR patches.

In paper(Gregor \& LeCun, 2010), the approach of train a neural network is inspired

$$
\text { 1:Repeat } Z=h_{(\alpha / \mathrm{L})}\left(Z-\frac{1}{L} W_{d}^{T}\left(W_{d} Z-X\right)\right)
$$

2:Until convergence; 3 :End function.

The elements of the eqn.are defined as:Filter matrix: $W_{e}=\frac{1}{L} D_{y}^{T}$.

Inhibition matrix: $\quad S=I-\frac{1}{L} D_{y}^{T} D_{y}$ Shrinkage $h_{\theta}(z)=\operatorname{sign}(z)(|z|-\theta)_{+}$.

function:

Here, $\mathrm{L}$ is a constant, which is larger than the largest eigenvalue of $D_{y}^{T} D_{y}$, function $h_{\theta}(z)$ is a component-wise shrinkage

$$
E(\mathrm{w})=\frac{1}{N} \sum_{i=1}^{N} C\left(\mathrm{~W}, \mathrm{y}_{i}\right) \text {, with } \mathrm{C}\left(\mathrm{W}, \mathrm{y}_{i}\right)=\frac{1}{2}\left\|z_{i}-f_{e}\left(W, y_{i}\right)\right\|_{2}^{2}
$$

Here, zi is the accurate sparse coefficient of yi which from traditional sparse method. And $f_{e}\left(W, y_{i}\right)$ is the transformation of eqn. . by the iterative shrinkage-thresholding algorithm (ISTA), The outline of ISTA is described in algorithm 3

Algorithm 3 ISTA

Require: L>largest eigenvalue of $W_{d}^{T} W_{d}$. Initialize: $\mathrm{Z}=0$.

function and $\theta$ is usually set as $\lambda / L$. In practice, because of this algorithm have to solve thousands of optimization and take tens of iterations to convergence, it' $\mathrm{s}$ too slow for practical applications. We use for reference paper (26), proposed a new idea which only need a fixed number of iteration $T$, they do not use fixed parameter in ISTA. The algorithm through minimize the following energy function to get the parameter $\mathrm{W}=$ (We,S, $\theta$ ):

$$
C\left(\mathrm{~W}, \mathrm{y}_{i}, \mathrm{x}_{i}\right)=\frac{1}{2}\left\|z_{i}-f_{e}\left(W, y_{i}\right)\right\|_{2}^{2}+\frac{v}{2}\left\|D_{x} z_{i}-x_{i}\right\|_{2}^{2}
$$

This will work better to predict the HR image. The outline of LISTA is describe in algorithm 4 and algorithm 5 .

Algorithm 4

Require : We, X.Initialize: $\mathrm{B}=\mathrm{WeX}$; $\mathrm{Z}(0)=\mathrm{h} \quad \theta \quad(\mathrm{B})$.Arguments are passed by reference.Variables $Z(t), C(t)$ and $B$ are saved for bprop.

$1:$ For $\mathrm{t}=1$ to $\mathrm{T}$ do; $2: \mathrm{C}(\mathrm{t})=\mathrm{B}+\mathrm{SZ}(\mathrm{t}-1)$;

$3: Z(t)=h \theta(C(t)) ; \quad 4:$ End for;5:Return $\mathrm{Z}=\mathrm{Z}(\mathrm{T})$

Algorithm 5

Require: $Z^{*}$ (accurate sparse coefficient from traditional methods); $\mathrm{Z}(\mathrm{t}), \mathrm{C}(\mathrm{t}), \mathrm{B}$ (saved in fprop).

Initialize:

$\delta B=0 ; \delta S=0 ; \delta \theta=0 ; \delta Z(\mathrm{~T})=\left(\mathrm{Z}(\mathrm{T})-Z^{*}\right)$
Extensively, in our super-resolution algorithm we rewrite the energy function as: 
2.1 Comparison with joint dictionary learning We compare our algorithm with Bicubic interpolation in visual result and other index. In order to make the fair comparison, we adopt the same training set and initial D for both methods.

a) Visual result: fig. 1(a)-(d) compared the results of Bicubic interpolation method with our fast image super-resolution four test images. From the left to right are the original HR images, the reconstructed images by Bicubic interpolation method and the reconstructed images by fast super-resolution method. From the results, we can easily see that our recovery are closed to the original images, the edge is more sharper than the Bicubic recovery, such as the moustaches of the cat, the texture of the freckles of the girl, the texture of the leaves and the hair of lena.
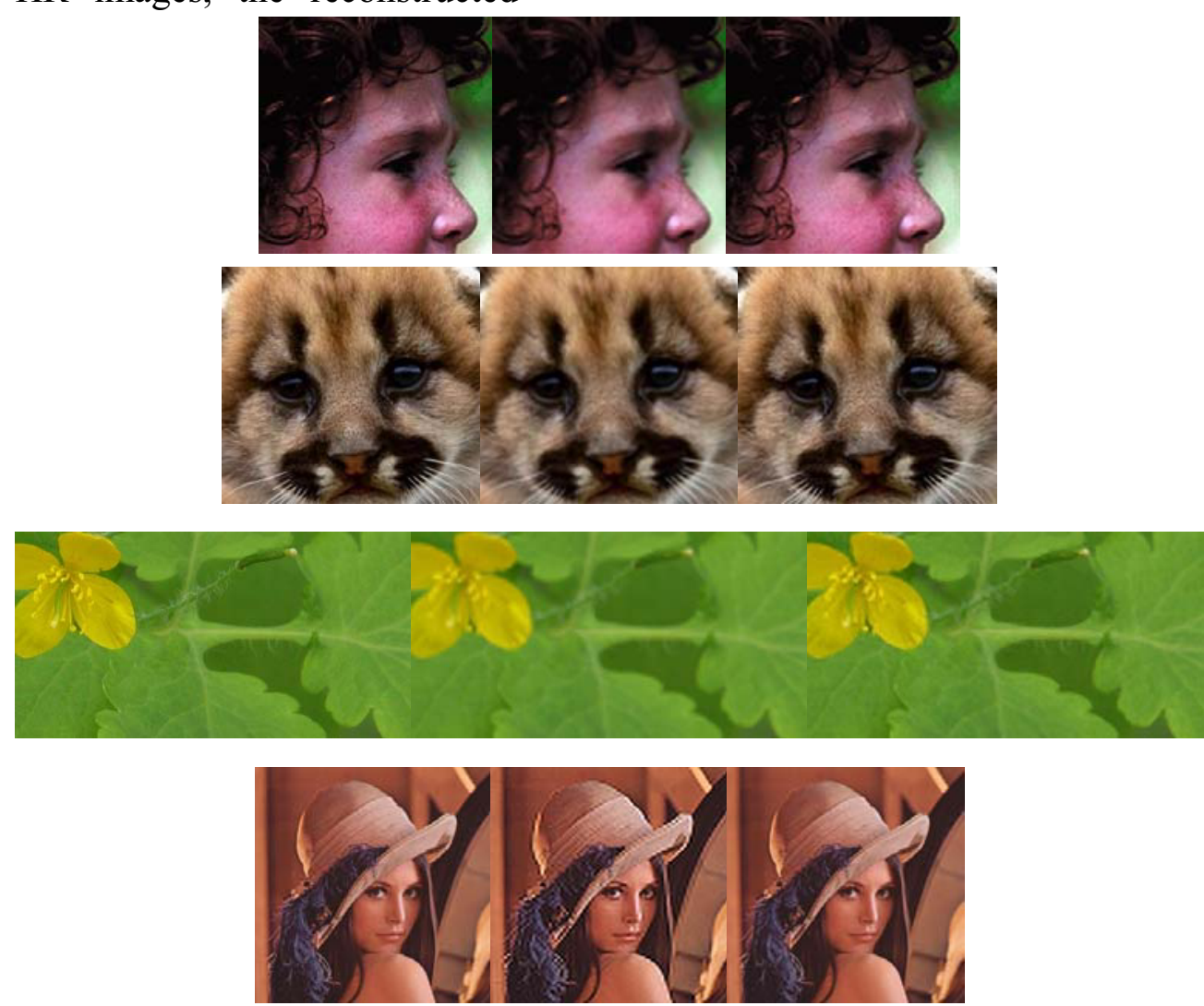

Fig. 1 from left to right: original image, Bicubic interpolation recovery, our recovery

b) Numerical result: now we compute the most easily defined via the mean squared Peak signal-to-noise ratio (PSNR) of the error (MSE), MSE is defined as: fast super-resolution image. PSNR is

$$
M S E=\frac{1}{M N} \sum_{m=0}^{M-1} \sum_{n=0}^{N-1}[\mathrm{I}(\mathrm{m}, \mathrm{n})-\mathrm{f}(\mathrm{m}, \mathrm{n})]^{2}
$$

Here, $\mathrm{I}$ and $\mathrm{K}$ are respectively represent the The PSNR is defined as: original image and our recovery.

$$
P S N R=10 \log _{10}\left(\frac{M A X_{I}^{2}}{M S E}\right)=20 \log _{10}\left(\frac{M A X_{I}}{\sqrt{M S E}}\right)=20 \log _{10}\left(M A X_{I}\right)-10 \log _{10}(\sqrt{M S E})
$$

Here, MAXI is the maximum possible pixel value of the image. When the pixels are represented using 8 bits per sample, this is
255. Typical values for the PSNR in lossy image and video compression are between 30 and $50 \mathrm{~dB}$, where higher is betters. 


\begin{tabular}{cccc}
\hline \multirow{2}{*}{ images } & Bicubic & \multicolumn{2}{c}{ Fast recovery } \\
\cline { 3 - 4 } & & $\mathrm{T}=3$ & $\mathrm{~T}=5$ \\
\hline girl & $31.699140 \mathrm{db}$ & $33.324861 \mathrm{db}$ & $33.336294 \mathrm{db}$ \\
cat & $28.3597 \mathrm{db}$ & $28.8539 \mathrm{db}$ & $28.8669 \mathrm{db}$ \\
leave & $37.197505 \mathrm{db}$ & $38.060257 \mathrm{db}$ & $38.060146 \mathrm{db}$ \\
lena & $32.794678 \mathrm{db}$ & $35.02261 \mathrm{db}$ & $35.022206 \mathrm{db}$ \\
\hline
\end{tabular}

Table I the PSNR of Bicubic interpolation recovery and our recovery

Table 1 shows the PSNR of test images. From table I we can see that our recovery outperforms the Bicubic interpolation recovery well with high PSNR. When the iteration times is fixed as 3 , the fast sparse coding is converging to optimal.

2.2 Algorithm efficiency

\begin{tabular}{ccccc}
\hline iamge & mesuares & Bicubic & Yang at el. & Fast recovery \\
\hline Girl & PSNR $(\mathrm{db})$ & 31.699140 & 33.34565 & 33.336294 \\
$254 \times 256$ & Time $(\mathrm{s})$ & 0.012371 & 285.496759 & 34.6597 \\
Cat & PSNR $(\mathrm{db})$ & 28.3597 & 28.8658 & 28.8669 \\
$326 \times 298$ & Time $(\mathrm{s})$ & 0.017776 & 446.296511 & 48.6235 \\
Leave & PSNR $(\mathrm{db})$ & 37.197505 & 38.0611 & 38.060146 \\
$328 \times 170$ & Time $(\mathrm{s})$ & 0.012091 & 246.902859 & 41.3629 \\
statuary & PSNR $(\mathrm{db})$ & 26.0253 & 26.3064 & 26.2923 \\
$256 \times 162$ & Time $(\mathrm{s})$ & 0.007996 & 180.382172 & 23.26 \\
\hline
\end{tabular}

Table II processing time of different SR method
In this subsection, we evaluate the efficiency of our algorithm and other state-of-art algorithms (Glasner, Bagon, \& Irani, 2009; Yang et al., 2010). All of the experiments are conducted in Matlab 8.1. Table II show the consumed times of different method. 
[3] Bousquet, O., \& Bottou, L. (2007). The Tradeoffs of Large Scale Learning. Paper presented at the Advances in Neural Information Processing Systems.

[4] Candes, E. J., Romberg, J. K., \& Tao, T. (2006). Stable signal recovery from incomplete and inaccurate measurements. Communications on Pure And Applied Mathematics, 59(8), 1207-1223. doi: Doi 10.1002/Cpa.20124

[5] Daubechies, I., Defrise, M., \& De Mol, C. (2004). An iterative thresholding algorithm for linear inverse problems with a sparsity constraint. Communications on Pure And Applied Mathematics, 57(11), 1413-1457. doi: Doi 10.1002/Cpa.20042 